\title{
LA NECESIDAD DE UN CAMBIO METODOLÓGICO EN LA ENSEÑANZA DEL EQUILIBRIO QUÍMICO: LIMITACIONES DEL PRINCIPIO DE LE CHATELIER
}

\author{
QUílLEZ PARDO, J.', SOLAZ PORTOLES, J.J.', CASTELLÓ HERNÁNDEZ, M. ${ }^{3}$ y SANJOSÉ LÓPEZ, V. ${ }^{4}$ \\ 'IB José Ballester. Valencia. \\ ${ }^{2}$ IB Lliria. Valencia. \\ ${ }^{3}$ Ext. IB Aldaia. Valencia \\ ‘ Área de Didáctica de las Ciencias Experimentales. Universitat de València.
}

\begin{abstract}
SUMMARY
This paper studies the didactic approach used in teaching Le Chatelier's Principle, showing that its usual formulation is ambiguous, and reports the way Chemistry textbooks use this principle in some specific cases with no mention of its limitations. The second part of this work analyses the strategies carried out by chemistry teachers when dealing with a specific equilibrium problem. It is shown how the usual methodology practised in both cases may lead to very important misconceptions, so a new methodological performance is proposed to achieve a real significative learning.
\end{abstract}

\section{INTRODUCCIÓN}

El aprendizaje del equilibrio químico requiere el conocimiento previo de un gran número de conceptos relacionados con él y la naturaleza abstracta del mismo hace que, según diversos autores (Wheeler y Kass 1978, Shayer y Adey 1984), requiera la utilizacion de pensamiento formal (Inheler y Piaget 1972) para su compren. sion. Todo ello hace que el equilibrio químico sea uno de los aspectos que mayor dificultad presenta para los estudiantes (Finley et al. 1982) y que su estudio no se inicie hasta los últimos cursos del bachillerato, una vez estudiada la reacción química y sus aspectos cuantitativos.

Normalmente, los alumnos no tienen esquemas conceptuales desarrollados sobre el equilibrio químico al comenzar su estudio, pero se ha observado que durante el desarrollo de los temas relacionados con este importante concepto aparecen una serie de dificultades de aprendizaje y de errores conceptuales de gran persistenciá, difíciles de eliminar (Pozo et al. 1991).

En la mayoría de los casos, no debemos considerar estos errores conceptuales como ideas espontáneas, sino como ideas inducidas a través de la enseñanza (Johnstone, MacDonald y Webb 1977, Hackling y Garnett 1985). El desarrollo de estos errores se debe, en muchos casos, a las analogías que tanto los profesores como los libros de texto usan para enseñar el concepto de equilibrio químico (Maskill y Cachapuz 1989) y están asociados, en algunas ocasiones, a problemas de lenguaje (Bergquist y Heikkinen 1990), al establecer los estudiantes asociaciones y analogías con los conceptos usados en física y en la vida diaria (Gussarsky y Gorodestky 1990).

Otra causa de las dificultades encontradas en el estudio del equilibrio químico está relacionada con la defíciente resolución de problemas (Selvaratnam y Frazer 1982, Camacho y Good 1989), debido, en gran parte, a que los alumnos to realizan un planteamiento previo acerca de lo que hay que resolver y a que carecen de estrategias adecuadas de resolución, limitándose, en la mayoría de los casos, a establecer relaciones entre todos los datos que se proporcionan en el problema y las ecuaciones que de forma necesaria creen que deben emplear. Las carencias y dificultades que indican estos autores guardan un paralelismo con otros estudios sobre resolución de problemas en Física y Química en los que se señalan, entre otras, los siguientes causas en el fracaso de los estudiantes en la resolución de problemas:

a) La falta de reffexión cualitativa previa y un tratamiento superficial del problema, que no se detiene en la 
clarificación de los conceptos (Nurrembern y Pickering 1987) y que conduce a un operativismo mecánico y a la no realización de un análisis de los resultados (Gil, Martínez y Senent 1988).

b) La dificultad en cuanto al control de variables (Selvaratnam y Kumarasinghe 1991) y en la relación de conceptos (Kempa 1986), lo que les impide la justificación de sus respuestas.

c) La incapacidad para distinguir entre la información que es esencial para resolver el problema y la que es irrelevante para ello (Kempa 1986).

Dada la importancia que el equilibrio químico tiene para el estudio de aspectos tan importantes como el comportamiento ácido-base, las reacciones de oxidación-reducción o las reacciones de precipitación, se hace necesario el conocimiento de las dificultades de aprendizaje y de los errores conceptuales relacionados con el mismo, así como su posible origen (Hierrezuelo y Montero 1989, Pozo et al. 1991), de forma que se puedan establecer estrategias didácticas para superarlos, facilitando con ello el estudio posterior de otros conceptos químicos íntimamente relacionados.

Uno de los aspectos más relevantes del estudio del equilibrio químico resulta ser el tratamiento de los factores relacionados con los desplazamientos que puede sufrir un sistema en equilibrio. En estos casos, para la predicción cualitativa de la evolución del sistema, suele jugar un papel fundamental el principio de Le Chatelier.

\section{EL PRINCIPIO DE LE CHATELIER}

En 1884, Henri Louis Le Chatelier, un químico francés, concibió un principio unifícado que describe cómo responden los sistemas en equilibrio ante un cambio en las condiciones que lo mantienen, y que reproducimos a continuación de forma literal (Gold y Gold 1984):

«Tout système en equilibre chimique stable soumis à I'influence d'une cause extérieure qui tend à faire varier soit sa température, soit sa condensation (pression, concentration, nombre de molecules dans l'unité de volume) đans sa totalité ou seulement dans quelques-unes des ses parties, ne peut éprouver que des modifications intérieures, qui, si elles se produisaient seules, amèneraient un changement de température ou de condensation de signe contraire à celui resultant de la cause extérieure.»

Este principio es conocido también como principio de Le Chatelier-Braun, debido a las aportaciones que Braun realizzó unos años más tarde. Mientras Le Chatelier describió el principio como "puramente experimental» (Paty 1985), Braun presentó una justificación teórica formal (Treptow 1980, Gold y Gold 1985) y, aunque en algunos aspectos su versión es más notable que la de Le Chatelier, adolece de las mismas debilidades, de las que nos ocuparemos más adelante.

En posteriores trabajos, Le Chatelier reformuló su principio de una forma más breve y generalizada, y que se aproxima a las versiones actuales que de este principio se dan en los libros de texto. Así, en el año 1888 (Gold y Gold 1985) y posteriormente en su libro Leçons sur le carbone, la combustion et les lois chimiques (Le Cha* telier 1908), lo estableció como sigue:

«Tout système en équilibre chimique éprouve, du fait de la variation d'un seul des facteurs de l'équilibre, une transformation dans un sens tel que, si elle se produisait seule, elle amènerait une variation du signe contraire du facteur consideré.»

El principio de Le Chatelier produjo un gran impacto en la química de fin del siglo pasado y la posibilidad de numerosas aplicaciones le proporcionó una popularidad que continúa hasta nuestros días (De Heer 1957).

Sin embargo, ya en 1909, los trabajos de P. Ehrenfert y de M.C. Raveau (De Heer 1957, Gold y Gold 1984) señalan la formulación vaga e imprecisa del principio de Le Chatelier, de forma que su aplicación literal puede conducir a resultados contradictorios y se insta, por primera vez, a una formulación más precisa basada en los principios de la termodinámica. A pesar de que estos primeros trabajos fueron desarrollados por otros autores durante las siguientes décadas, sus contribuciones callsaron un impacto mínimo. Así, De Heer (1957) señala que muchos científicos tienen la idea errónea de que el principio de Le Chatelier es «verdadero» en el sentido de que expresa una ley fundamental de la naturaleza, cuya validez no puede ser cuestionada.

Más recientemente, Treptow (1980), Allsop y George (1984) y Gold y Gold $(1984,1985)$ vuelven a insistir en que el principio es vago y ambiguo -sugiriendo que sea reemplazado por una aproximación didáctica a partir de las leyes de van't Hoff del equilibrio- y Bridgart y Kemp (1985) han mostrado nuevos ejemplos acerca de las limitaciones en el uso del mismo. Por otro lado, Mazo y Barnhard (1972), Bodner (1980), Fernández-Prini (1982), Brice (1983) y Treptow (1984) discuten los resultados erróneos a los que conduce la aplicación del principio de Le Chatelier en la predicción de la variación de la solubilidad debido a un cambio de temperatura.

Uno de los ejemplos más empleados por la bibliografía actual (aunque debemos hacer notar que los primeros artículos hace más de treinta años que se publicaron), para mostrar «la falsedad» del principio de Le Chatelier, es el análisis de la síntesis del amoníaco (De Heer 1957, Katz 1961, Wright 1969, Levine 1981, Helfferich 1985). Así, si consideramos el equilibrio representado por la siguiente ecuación:

$$
\mathrm{N}_{2}(\mathrm{~g})+3 \mathrm{H}_{2}(\mathrm{~g}) \rightleftharpoons 2 \mathrm{NH}_{3}(\mathrm{~g})
$$

y nos preguntamos qué ocurrirá si añadimos al sistema, a presión y temperatura constantes, una cierta cantidad de nitrógeno, podemos responder de una forma superficial, basándonos en la aplicación mecánica del principio de Le Chatelier, que el sistema «reaccionará» oponiéndose a esa adición, desplazándose el equilibrio hacia la derecha, con formación de una mayor cantidad de amoníaco. Sin embargo, si se realiza un análisis exhaustivo, 
se comprobará que el propio principio no nos informa de la dirección en la cual se desplaza el equilibrio. En este sentido, debemos tener presente que el proceso se produce a presión constante, de forma que la adición de una cierta cantidad de nitrógeno produce un aumento en su presión parcial, al mismo tiempo que disminuye la presión parcial del hidrógeno. Por tanto, se producen dos variaciones que producen efectos contrapuestos, por lo que, en principio, no podemos predecir la dirección en la que se desplazará el equilibrio.

Un estudio teórico cuantitativo de este equilibrio (De Heer 1957, Katz 1961, Wright 1969, Helfferich 1985) demuestra que lo que ocurra dependerá de la composición inicial de la mezcla en equilibrio a la que se añade nitrógeno. Si la fracción molar del nitrógeno es menor que 0,5 , el equilibrio se desplaza formando más amonía. co y, si su fracción molar es mayor que 0,5 , el amoníaco se descompone para formar más nitrógeno. Para otras reacciones químicas, se pueden establecer sendas relaciones cuantitativas (Katz 1961) a ia hora de predecir la dirección en la que se desplazará un sistema en equilibrio por la adición al mismo -a temperatura y presión constantes - de una cierta cantidad de un reactivo o de un producto. Así, para un equilibrio que podemos representar como:

$$
\mathrm{a} \mathrm{A}(\mathrm{g})+\mathrm{b} \mathrm{B}(\mathrm{g}) \rightleftharpoons \mathrm{p} C(\mathrm{~g})+\mathrm{q} \mathrm{D}(\mathrm{g})
$$

y definido $\Delta n=(p+q)-(a+b)$, se puede establecer, por ejemplo, que, si se añade un producto $\mathrm{C}$, el equilibrio se desplaza hacia la formación de una mayor cantidad de productos si $X_{\mathrm{C}}>\mathrm{p} / \Delta \mathrm{n}$ (donde $X_{\mathrm{C}}$ es la fracción molar inicial de $\mathrm{C}$ en el equilibrio).

\section{OBJETIVOS}

El principio de Le Chatelier ha sido objeto de estudio por una gran cantidad de autores con el propósito de estudiar las dificultades y los errores conceptuales relacionados con el mismo (Driscol 1960, Johnstone, McDonald y Webb 1977, Wheeler y Kass 1978, Felipe 1981, Pereira 1981, Hackling y Garnett 1985, Gorodetsky y Gussarsky 1986, Gil 1988, Bergquist y Heikkinen 1990, Banerjee 1991). A pesar de ello, uno de los aspectos menos estudiados han sido los errores conceptuales asociados con las limitaciones señaladas de este principio (Driscoll 1960, Johnstone et al. 1977).

Pensamos que el mismo planteamiento didáctico del principio de Le Chatelier puede ser origen de importantes errores conceptuales. Por ello, nuestro estudio se centrará en cómo se enseña este principio a partir de un análisis de la formulación que del mismo se realiza en los libros de texto y de cómo éstos lo utílizan para realizar predicciones cualitativas acerca de la evolución de un sistema en equilibrio que ha sido perturbado, para establecer, posteriormente, las relaciones existentes entre estos planteamientos didácticos $\mathrm{y}$ la forma en que los profesores utilizan este principio en el aula para resolver problemas de equitibrio químico.

Según este planteamiento previo, formulamos para este trabajo los siguientes objetivos:

1. Analizar la forma en que los libros de texto estudian Ios factores que afectan a un sistema en equilibrio.

2. Establecer la forma en que el principio de Le Chatelier se enuncia en los libros de texto y cómo se utiliza en la predicción de la evolución de un sistema en equilibrio que ha sido perturbado.

3. Comprobar si el citado principio se establece como principio infalible o si se señalan sus limitaciones.

4. Estudiar cómo aplican los profesores este principio, como estrategia didáctica, en la resolución de problemas.

\section{HIPÓTESIS DE TRABAJO}

A pesar de la abundante bibliografía que discute la formulación ambigua y la supuesta validez del principio de Le Chatelier, pensamos que éste sigue presentándose tanto por los profesores como por los libros de texto de una forma simplificada, sin hacer referencia explícita a sus limitaciones, pot to que puede aparecer como un principio infalible que prediga, en cualquier caso, la evolución de un sistema en equilibrio. Esta presentación puede llevar a los alumnos a una aplicación mecánica y superficial del mismo, en la que no realicen el pertinente control de variables y no utilicen la expresión de la constante de equilibrio para apoyar sus predicciones.

Podemos concretar las hipótesis de las que partimos en los siguientes puntos:

Hipótesis 1. En los libros de texto, el principio de Le Chatelier se formula generalmente de una forma simplificada, sin fundamentación teórica, y sigue utilizándose como guía infalible en la predicción de la evolución de un sistema en equilibrio por la variación de las magnitudes que lo definen.

Hipótesis 2. En función de esta formulación, la forma en la que los profesores resuelven problemas en los que se aplica este principio conduce a un tratamiento superficial del mismo, en el que predomina un operativismo mecánico y una ausencia del control de las variables que intervienen en el sistema.

\section{METODOLOGÍA}

Los libros de texto empleados para realizar el análisis establecido corresponden a manuales de Química General de los niveles de COU y primero de universidad, por 
ser en éstos donde se introducen, de forma general, los aspectos relacionados con el equilibrio químico. En consonancia con los objetivos planteados y las hipótesis formuladas, se elaboró el siguiente cuestionario:

\section{Cuestionario para el análisis de textos}

1. ¿Se formula el principio de Le Chatelier para prever los desplazamientos del equilibrio químico?

2. ¿Se utiliza la expresión de la constante de equilibrio para el mismo fin?

3. ¿Se menciona algún tipo de justificación del principio: teórica (termodinámica), empírica o inductiva, osimplemente sólo se menciona?

4. ¿Se emplea dicho principio para predecir la respuesta del sistema en equilibrio a cambios de temperatura, de concentración y de presión (volumen)?

5. ¿Se analiza la posible respuesta del sistema en equilibrio por la presencia de un catalizador o la adición de un gas inerte o un sólido (en este caso, en equilibrios heterogéneos)?

6. ¿Se especifica que, cuando se modifique una variable del equilibrio y se aplique el principio de Le Chatelier en la predicción de la evolución đel sistema, se deben controlar las restantes variables que lo definen?

7. ¿Se previene respecto a los posibles casos en tos que la aplicación del principio proporcione resultados contradictorios o de incumplimiento del mismo?

Por otro lado, la muestra de profesores que participaron en esta investigación corresponde a un total de 26 , de los cuales 19 son de enseñanza secundaria y 7 de nivel universitario, la mayoría de ellos encargados de impartir un curso de Química General de su nivel correspondiente. A todos ellos se les proporcionó el siguiente problema:

El principio de Le Chatelier puede presentar en su introducción y aplicación algunas dificultades para los estudiantes de Química.

Indica la forma que creas más conveniente para desarrollar en clase el problema que presentamos a continuacion, con objeto de estudiar el principio de Le Chatelier, en este caso concreto, haciendo todos los comentarios que creas conveniente acerca de la idoneidad del problema para el cumplimiento del objetivo propuesto.

A un recipiente que contiene únicamente $\mathrm{H}_{2} \mathrm{~S}(\mathrm{~g})$ se añade una cantidad de $\mathrm{NH}_{4} \mathrm{HS}(\mathrm{s})$ de forma que se establece el equilibrio representado por la siguiente ecuación:

$$
\mathrm{NH}_{4} \mathrm{HS}(\mathrm{s}) \rightleftharpoons \mathrm{NH}_{3}(\mathrm{~g})+\mathrm{H}_{2} \mathrm{~S}(\mathrm{~g})
$$

EI análisis deI mismo, correspondiente a una temperatura de $25^{\circ} \mathrm{C}$, revela la siguiente composición de la mezcla gaseosa:

$$
\mathrm{n}\left(\mathrm{H}_{2} \mathrm{~S}\right)=1,65 \times 10^{-2} \mathrm{~mol} ; \quad \mathrm{n}\left(\mathrm{NH}_{3}\right)=1,10 \times 10^{-2} \mathrm{~mol}
$$

y se determina que el volumen que ocupan es de $1 \mathrm{~L}$.

I. Argumenta cualitativamente, de forma breve pero razonada, cómo se verá afectado el equilibrio anterior por la adición, a presión y temperatura constantes, de las sustancias que se indican en cada uno de los siguientes apartados:
a) $\mathrm{H}_{2} \mathrm{~S}(\mathrm{~g})$
b) $\mathrm{NH}_{4} \mathrm{HS}(\mathrm{s})$
c) $\mathrm{N}_{2}(\mathrm{~g})$

2. Si la cantidad añadida de $\mathrm{H}_{2} \mathrm{~S}(\mathrm{~g})$ en el apartado anterior es de $1,35 \times 10^{-2}$ mol, ¿cuál será la composición de la mezcla de gases cuando se alcance nuevamente el equilibrio?

Con el objeto de que cada profesor pudiese reflexionar el problema con el tiempo que considerase necesario, se pidió a los profesores que lo entregasen una vez lo hubiesen resuelto, sin establecer una fecha límite para ello.

El problema elegido plantea una situación semejante a la que describe la bibliografía para la síntesis del amoníaco en la que, por adición, a presión y temperatura constantes, de una de las sustancias gaseosas participantes en el equilibrio, la aplicación del principio de Le Chatelier conduce a resultados contradictorios. También se plantean otros dos apartados que suponen la adición de un gas inerte y de una mayor cantidad de sólido. En ambas situaciones se pueden presentar nuevas dificultades si se intentan relacionar estos efectos con el principio de Le Chatelier (Driscoll 1960), en lugar de realizar, como en el primer caso, un riguroso control de variables y una argumentación basada en la constante de equilibrio. De nuevo, en consonancia con los objetivos formulados y las hipótesis planteadas, se elaboró el siguiente cuestionario para el análisis del problema resuelto por cada uno de los profesores participantes en la investigación:

\section{En la parte cualitativa}

a) ¿Se realiza un análisis previo en el que se procede a un control de variables?

b) ${ }_{S}$ Se hace únicamente referencia al principio de Le Chatelier para indicar el posible desplazamiento del equilibrio?

c) ¿Se utiliza la expresión de la constante de equilibrio para fundamentar el posible desplazamiento del equilibrio?

d) ¿Se indica con total seguridad la dirección en la que se desplazará el equilibrio, en cada uno de los casos?

\section{En la resolución de la parte cuantitativa}

a) iSe realiza un control de variables haciendo referencia a la variación del volumen?

b) LSe indican posibles estrategias de resolucion y la conveniencia de emplear la desarrollada en el problema?

c) ¿Se procede directamente a resolver el problema, planteando las ecuaciones correspondientes?

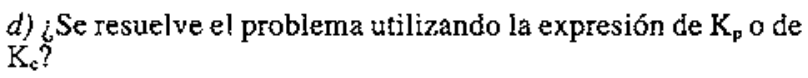

e) ¿El equilibrio se ha desplazado hacia la formacion de una mayor cantidad de productos?

f) ¿Se realiza un análisis de los resultados? 


\section{RESULTADOS}

El cuestionario referido al análisis de textos, realizado a partir dei estudio llevado a cabo para un total de 18 libros de Química de COU y 12 de Química General correspondientes a un primer curso universitario, proporciona los resultados que se reflejan en la tabla 1 , en la que los datos que se indican corresponden a los porcentajes de respuestas afirmativas para cada uno de los ítems.

Tabla I

Resultados del análisis de textos (\% de respuestas afirmativas).

\begin{tabular}{|c|c|c|}
\hline Item & $\begin{array}{c}(\mathrm{N}=18) \\
\mathrm{COU}\end{array}$ & $\begin{array}{c}(\mathrm{N}=12) \\
\text { Universidad }\end{array}$ \\
\hline $\begin{array}{l}1 \\
2 \\
3 \text { justificación teórica } \\
\text { justificacion empírica o inductiva } \\
\text { simple enunciado }\end{array}$ & $\begin{array}{r}100 \\
67 \\
11 \\
44 \\
45\end{array}$ & $\begin{array}{r}100 \\
92 \\
8 \\
42 \\
50\end{array}$ \\
\hline $\begin{array}{l}4 \text { cambios de presion (volumen) } \\
\text { cambios de temperatura } \\
\text { cambios de concentración }\end{array}$ & $\begin{array}{l}100 \\
100 \\
100\end{array}$ & $\begin{array}{l}100 \\
100 \\
100\end{array}$ \\
\hline $\begin{array}{l}5 \text { presencia de catalizador } \\
\text { adición de sólido } \\
\text { adición de gas inerte } \\
\text { a } V=\text { cte } \\
\text { a } P=\text { cte. }\end{array}$ & $\begin{array}{r}43 \\
0 \\
11 \\
0\end{array}$ & $\begin{array}{l}60 \\
17\end{array}$ \\
\hline $\begin{array}{l}6 \\
7\end{array}$ & $\begin{array}{r}11 \\
6\end{array}$ & $\begin{array}{r}8 \\
17\end{array}$ \\
\hline
\end{tabular}

Tabla 11

Resultados del anátisis deI problema: Parte cualitativa (\% de respuestas afirmativas). $(\mathrm{N}=24)$

\begin{tabular}{lccc}
\hline Item & $\mathrm{H}_{2} \mathrm{~S}(\mathrm{~g})$ & $\mathrm{NH}_{4} \mathrm{HS}(\mathrm{s})$ & $\mathrm{N}_{2}(\mathrm{~g})$ \\
\cline { 2 - 4 } & 21 & - & 46 \\
la & 30 & 0 & 0 \\
lc & 70 & 42 & 54 \\
ld izquierda & 75 & 4 & 21 \\
derecha & 4 & - & 29 \\
depende & 17 & - & 4 \\
no se indica & 4 & 96 & 46 \\
igual & - & &
\end{tabular}

\section{Tabla III}

Resultados del anásisis del problema: parte cuantitativa (\% de respuestas afirmativas). ( $\mathrm{N}=24)$

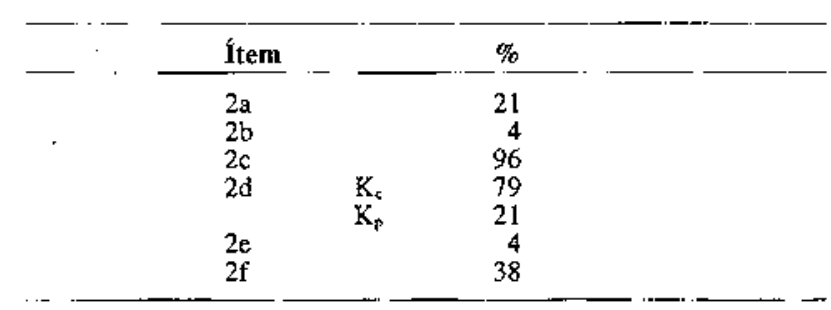

El cuestionario correspondiente al análisis del problema resuelto por los profesores y evaiuado para un total de 24 resoluciones completas proporciona los resultados que se reflejan en las tablas II y III. La tabla II corresponde a la parte cualitativa del problema, en la que se analizan las respuestas que se proporcionan para cada uno de los tres apatados de que consta; en la tabla III se reflejan los resultados correspondientes al análisis de la segunda parte del problema. En ambos casos, los datos que se indican corresponden al porcentaje de respuestas afirmativas de cada uno de los ítems.

\section{DISCUSIÓN}

A la vista de los resultados obtenidos en el análisis de textos (Tabla 1 ) y del correspondiente a la resolución del problema (Tablas II y III), realizaremos un breve comen* tario de los mismos.

\section{Análisis de textos}

Como puede apreciarse (item l) el principio de Le Chatelier es ampliamente utilizado, en ambos niveles, en la pređicción del desplazamiento del equilibrio químico cuando se modifica alguna variable que lo define. Ade más, la formulación del principio que aparece en los textos es muy concisa en la mayoría de las ocasiones, aproximándose su enunciado a las últimas formulaciones que realizó el autor, lo que muestra el escaso impacto que han tenido los intentos de enunciar el principio de Le Chatelier de una forma menos ambigua (Treptow 1980).

Los resultados correspondientes al item 2 muestran que un porcentaje elevado de libros ilustra la regla cualitati-

va (principio de Le Chatelier) con una discusión cuantitativa basada en la expresión de la constante de equilibrio en donde fundamentalmente se analiza la variación de la concentración de una de las sustancias que participan en la reacción por una adición o eliminación de una cierta cantidad de la misma. Esta aproximación, unida al escaso tratamiento que se realiza para equilibrios heterogéneos (item 5) puede provocar el error conceptual de que la adición o eliminación de una pequeña cantidad de sólido desplace el equilibrio en algún sentido (Driscoll 1960, Gorodetsky y Gussarsky 1986).

Los resultados del item 3 demuestran que el principio de Le Chatelier no se fundamenta sobre las bases de la termodinámica, sino que se prefiere ofrecer un principio surgido de un proceso de inducción a partir de múltiples experimentos o casos particulares.

En todos los textos se analizan los cambios de presión, temperatura y concentración sobre el sistema en equilibrio (item 4), empieando, normalmente, dos aproximaciones didácticas diferentes. La más utilizada es la que finaliza con la formulación de un principio general que engloba los casos particulares que previamente han sido discutidos (variaciones de concentración, presión y temperatura). En la otra aproximación, se realiza un plantea 
miento inverso al enunciar en primer lugar el principio y confirmarlo posteriormente mediante los tres casos mencionados anteriormente.

La introducción cinética que suele realizarse del equili* brio químico puede provocar algunos errores conceptuales y, en particular, los relacionados con el desplazamiento del equilibrio por la presencia de catalizadores (Johnstone et al. 1977, Hackling y Gamett 1985, Cachapuz y Maskill 1989). A pesar de ello, este hecho sólo se discute explícitamente en torno al $50 \%$ de los textos (item 5).

Otro aspecto escasamente tratado en los textos es el posible efecto que puede provocar sobre el sistema en equilibrio la adición al mismo de un gas inerte $(11 \%$ en los libros de Química de COU y 33\% en los de Química General), limitándose, en estos casos, a la adición a volumen constante (ítem 5).

Los resultados correspondientes al item 6 reflejan el tratamiento superficial que se realiza al considerar la perturbación del equilibrio para predecir su evolución: poquísimos libros (aproximadamente un 10\%) llevan a cabo un control de las variables implicadas, señalando qué variables permanecen constantes y cuáles se modifican.

Por último, puede apreciarse (ítem 7) que los autores rehuyen aquellos casos donde el principio de Le Chatelier no conduce a un pronóstico correcto o puede resultar ambiguo. El bajo porcentaje de respuestas afirmativas de este ítem puede dar idea de la imagen de "regla infalible» con la que presentan los diferentes manuales este principio.

Por otra parte, realizada la prueba $X^{2}$ (chi cuadrado) con los ítems de nuestro análisis de textos, se observó que no existen diferencias significativas entre el tratamiento realizado por los textos preuniversitarios y el de los universitatios.

\section{Análisis de la resolución del problema}

En este apartado, únicamente discutiremos los aspectos más relevantes, dejando para un trabajo posterior una discusión más exhaustiva y completa de los đatos que se proporcionan.

Analicemos los resultados de la tabla II para cada uno de los apartados de la primera parte del problema:

a) $\mathrm{H}_{2} \mathrm{~S}(\mathrm{~g})$. Sólo un $21 \%$ de las respuestas realiza un control de variables y, aunque un porcentaje del $30 \%$ argumenta únicamente en función del principio de Le

Chatelier y el resto empiea la expresión de la constante de equilibrio, sólo en un $17 \%$ de las respuestas se hacen predicciones correctamente fundamentadas.

b) $\mathrm{NH}_{4} \mathrm{HS}(\mathrm{s})$. En este caso no se establece ninguna relación con el principio de Le Chatelier y, aunque sólo un $42 \%$ utiliza de forma explícita la expresión de la constante de equilibrio, la mayoría de las respuestas son correctas (96\%). Sin embargo, debemos hacer notar que entre los comentarios que algunos profesores realizaban cuando se les mostraba el problema, y antes de proceder a su resolución, se indicaba con gran seguridad que en este caso se formarían más productos de reacción. A pesar de elio, en ningún caso se menciona en los comentarios escritos que esta aproximación superficial pueda originar errores conceptuales en los alumnos, como se desprende de los primeros resultados que los autores de este trabajo están obteniendo a este respecto.

c) $N_{2}(g)$. En este apartado, el porcentaje de respuestas donde se realiza un control de variables es superior al primer apartado, indicando que el volumen del reactor aumentará. Sin embargo, el número de argumentos basados en la expresión de la constante de equilibrio es inferior $(54 \%)$ y el porcentaje de respuestas correctas es también muy bajo (29\%). Es de destacar el elevado porcentaje correspondiente a la respuesta que indica que el equilibrio permanece inalterado $(46 \%)$, basando esta afirmación en el hecho de que la adición de esta sustancia no provoca ninguna reacción con las sustancias participantes en el equilibrio.

Finalmente, analicemos los resultados de la tabla III.

Destacaremos en esta discusión que la mayor parte de la resoluciones $(96 \%)$ inicia la segunda parte del problema sin realizar un análisis cualitativo previo y sin comentar las posibles estrategias de resolución a seguir, así como las ventajas e inconvenientes que pueden reportar.

El procedimiento empleado es generaimente de tipo algorítmico en el que se plantean directamente las ecuaciones y se procede a su resolución. En la mayor parte de los casos (79\%) se emplea la expresión de la constante de equilibrio referida a las concentraciones $\left(\mathrm{K}_{\mathrm{c}}\right)$, sin considerar que el volumen no permanece constante, en lugar de emplear la expresión de $\mathrm{K}_{\mathrm{p}}$, a partir đe la que, de una forma más sencilla, se pueđe hallar la solución correcta al problema (realizado únicamente por el $4 \%$ de los profesores).

El análisis de los resultados que proporciona el problema, a la luz de una discusión cualitativa previa, es de una gran riqueza. Esta etapa final únicamente es realizada por el $38 \%$ de los profesores, aunque, como ya se ha señalado, la mayoría parten de planteamientos incorrectos.

Finalmente, debemos hacer mención de dos comentarios remitidos por sendos profesores que no han podido ser analizados como en los otros veinticuatro casos, ya que no han procedido a resolver el problema en ninguna de sus partes y la reflexiones que realizaban son muy generales, sin mencionar las posibles dificultades que puede tener el problema en su resolución.

\section{CONCLUSIONES}

El estudio llevado a cabo en este trabajo indica el énfasis mostrado por los autores de libros de Química General en la formulación del principio de Le Chatelier, empleando para ello un enunciado de marcado carácter 
inductivo, que carece de fundamentación teórica y que no muestra sus limitaciones, y utilizándose como guía infalible en la predicción de la evolución de un sistema en equilibrio por la variación de las variables que lo definen.

En relación con esta presentación, la resolución por profesores de problemas de equilibrio químico, en los que puede tener aplicación el principio de Le Chatelier, conduce a un tratamiento superficial del mismo en el que no se realiza un control riguroso de variables, empleándose estrategias de resolución de tipo algorítmico que no cuestionan su validez y que finalmente lleva a la obtención de un resultado que en muy pocos casos es analizado.

La confirmación de las dos hipótesis formuladas indica que las dificultades que pueden encontrar los alumnos, al estudiar los conceptos del equilibrio químico y los errores conceptuales que pueden aparecer, guardan una estrecha relación con la forma en que estos conceptos se enseñan, al centrarse los mismos en un aprendizaje memorístico y repetitivo en el que no se favorece el pensamiento divergente, produciendo con ello aprendizajes carentes de significado químico.

El escaso impacto que ha tenido en los textos y en los profesores de química Ia gran cantidad de trabajos que

\section{REFERENCIAS BIBLIOGRÁFICAS}

ALLSOP, R.T. y GEORGE, N.H., 1984. LeChatelier--a redundant principle?, Ed. Chem, march, pp. 54-56

BANERJEE, A.C., 1991. Misconceptions of students and teachers in chemical equilibrium, Int. $J$. Sci. Educ., Vol. 13 (4), pp. 487-494.

BANERJEE, A.C. y POWER, C.N., 1991. The development of modules for the teaching of chemical equilibrium, Int. J. Sci. Educ., Vol. 13 (3), pp. 355-362.

BERGQUIST, W. y HEIKKINEN, H., 1990. Student Ideas Regarding Chemical Equilibrium, J. Chem. Ed., Vol. 67 (12), pp. 1000-1003.

BODNER, G.M., 1980. On the Misuse of Le Chatelier's Principle for the Prediction of the Temperature Dependence of the Solubility of Salts, J. Chem. Ed., Vol. 57 (2), pp. 117-119.

BODNER, G.M., 1986. Constructivism: A Theory of Knowledge, J. Chem. Ed., Vol. $63(10)$, pp. 873-878.

BRICE, L.K., 1983. Le Chatelier's Principle: The Effect of Temperature on the Solubility of Solids in Liquids, J. Chem. Ed., Vol. 60 (5), pp. 387-389. cuestionan el principio de Le Chatelier apunta la necesidad de acercar la investigación educativa a la realidad del proceso educativo (Banerjee y Power 1991). Este conocimiento no es suficiente para evitar inducir errores o superar dificultades, sino que, desde una perspectiva constructivista (Bodner 1986, Novak 1988, Saunders 1992) y dentro del marco curricular (Caamaño 1988), las estrategias didácticas que se utilicen deben emplear una metodología (Gil et al. 199I) que propicie un verdadero aprendizaje significativo.

Ello puede conseguirse, en este caso concreto, a partir del correcto control de variables (Linn et al. 1989), mediante la utilización de la expresión de la constante de equilibrio (Katz 1961) en la predicción de la evolución de un sistema en equilibrio por variación de la presión (o del volumen), la adición o sustracción de una de las sustancias que participan en el equilibrio (Castelló y Qúlez 1992) o de un gas inerte (Quílez 1988) y de su discusión cuantitativa, incluyendo variaciones de temperatura, a partir del estudio de las leyes de la termodinámica (Katz 1961, Gold y Gold 1984, Kemp 1987, Solomon 1991, Solaz 1992). De esta forma se evita, además, transmitir una imagen de la ciencia -que trasciende de la presentación usual del principio de Le Chatelier-basada en las ideas de Bacon y Pearson, que siguen apareciendo en muchos libros de texto (Novak 1984)
BRIDGART, G.J. y KEMP, H.R., 1985. A Limitation on the Use of the Le Chatelier's Principle, The Australian Science Teachers' Journal, Vol. 31, pp. 60-62.

CAAMAÑO, A., 1988. Tendencias actuales en el curículo de ciencias, Enseñanza de las Ciencias, 6 (3), pp. 265-277.

CACHAPUZ, A.F.C. y MASKILL, R., 1989. Using word association informative classroom test: following the learning of Le Chatelier's principle, Int.J.Sci.Educ, Vol. 21 (2), pp. 235-246.

CAMACHO, M. y GOOD, R., 1989. Problem Solving and Chemical Equilibrium: Succesful versus Unsuccesful Performance, Journal of Research in Science Teaching, Vol. 26 (3), pp. $251-272$.

CASTELLO, M. y QUfLEZ, J., 1992. La construcción de la Química con ayuda delordenador. (Consellería de Educación: Valencia)

DE HEER, J., 1957. The Principle of Le Chatelier and Braun, J. Chem. Ed., Vol. 34 (8), pp. 375-380.

DRISCOLL, D.R., 1960. The Le Chatelier Principle, Australian Science Teachers Journal, Vol. 6 (3), pp. 7-15. 
FELIPE, E., 1981. Estudio del equilibrio químico homogéneo para un currículo de Química - un problema didáctico. Tesis doctoral. Universidad de Valladolid.

FERNANDEZ-PRINI, R, 1982. Le Chatelier's Principle and the Prediction of the Effect of Temperature on Solubilities, J. Chem, Ed., Vol. 59 (7), pp. 550-553.

FINLEY, F.N., STEWART, J, y YARROCH, W.L., 1982. Teachers' perceptions of important and difficult science content, Science Education, Vol. 66 (4), pp. 531-538.

GlL, V.M.S., 1988. University students' assessment of the explanatory content of justification statements, Int. J. Sci. Educ., Vol. 10 (5), pp. 581-588.

GIL, D., MARTÍNEZ, J. y SENENT, F., 1988. El fracaso en la resolucion de problemas de Física: una investigación orientada por nuevos supuestos, Enseñanza de las Ciencias, 6(2), pp. 131-146.

GIL, D., CARRASCOSA, J, FURIO, C. y MARTÍNEZ, J, 1991. La Enseñanza de las Ciencias en la Educación Secundaria. (ICE-Horsori: Barcelona).

GOLD, I. y GOLD, V., 1984. Neither Le Chatelier's nor a Principle?, Chemistry in Britain, September, pp. 802-804.

GOLD, J. y GOLD, V., 1985. Le Chatelier's Principle and the Laws of Van't Hoff, Ed. Chem., may, pp. 82-85.

GORODETSKY, M. y GUSSARSKY, E., 1986. Misconceptualization of the chemical equilibrium concept as revealed by different evaluation methods, Eur.J. Sci. Educ., Vol. 8 (4), pp. 427-441

GUSSARSKY, E. y GORODETSKY, M., 1990. On the concept achemical equilibriums: The associative framework, Journal of Research in Science Teaching, Vol. 27 (3), pp. 197-204.

HACKLING, M.W. y GARNETT,P.J., 1985. Misconceptios of Chemical Equilibrium, Eur. J. Sci. Educ., Vol. 7 (2), pp. 205-214

HELFFERICH, F.G., 1985. Le Chatelier, - Right or Wrong? Optimizing Chemical Reaction Equilibra, J. Chem. Ed., Vol. $62(4), 305-308$.

HIERREZUELO, J. y MONTERO, A., 1989. La ciencia de los alumnos. Su utilización en la didáctica de la Física y la Química. (Laia/MEC: Barcelona).

INHELER, B. y PIAGET, J., 1972. De la lógica del niño a la lógica del adolescente. (Paidós: Buenos Aires).

JOHNSTONE, A.J., MACDONALD, J.J. y WEBB, G., 1977. Chemical equilibrium and its conceptual difficulties, Ed.Chem., Vol. 14, pp. 169-171.

KATZ, L., 1961. A Systematic Way to Avoid Le Chatelier's Principle in Chemical Reactions, J. Chem. Ed., Vol. 38 (7), pp. 375-377.

KEMP, H.R., 1987. The Effect of Temperature and Pressure on Equilibria: A Derivation of the Van't Hoff Rules, $J$. Chem. Ed., Vol. 64 (6), pp. 482-484.

KEMPA, R.F., 1986. Resolución de problemas de Química y estructura cognoscitiva, Enseñanza de las Ciencias, 4(2), pp. 99-110.

LE CHATELIER, H.L., 1908, Lecons sur le carbone, la combustion et les lois chimiques. (Dunod et Pinat Editeurs: París).

LEVINE, I.N,, 1981. Fisicoquimica. (McGraw-Hill: Bogota).
LINN, M.C., CLEMENT, C., PULLOS, S. y SULLIVAN, P.; 1989. Scientific Reasoning During Adolescence: The Influence of Instruction in Science Know ledge and Reasoning Strategies, Journal of Research in Science Teaching, Vol. 26 (2), pp. 171-187.

MASKILL, R. y CACHAPUZ, A.F.C., 1989. Learning about the chemistry topic of equilibrium: the use of word association tests to detect developing conceptualizations, Int.J.Sci.Educ., Vol. 11 (1), pp. 57-69.

MAZO, R.M. y BARNHARD, R., 1972. The Solubility of Sodium Acetate in Water and Le Chatelier's Principle, $J$. Chem. Ed., Vol. 49 (9), pp. 639-640.

NOVAK, J.D., 1984. Application of Advances in Learning Theory and Philosophy of Science in the Improvement of Chemistry Teaching, J. Chem. Ed., 61(7), pp. 607-612.

NOVAK, I.D., 1988. Constructivismo humano: un consenso emergente, Enseñanza de las Ciencias, 6(3), pp. 213-223.

NURRENBERN, S.C., y PICKERING, M., 1987. Concept Learning versus Problem Solving: Is There a Difference?, $J$. Chem. Ed., Vol, 64 (6), pp. 508-510.

PATY, M., 1985. Le Chatelier y la ley de los equilibrios químicos, Mundo científico, Vol. 5 (49), pp. 808-810.

PEREIRA, M.P.B.A., 1981. Teaching and learning difficulties in chemical equilibrium in secondary schools in Portugal. Tesis doctoral. Universidad de East Anglia.

POZO, J.I., GOMEZ, M.A, LIMÓN, M. y SANZ, A., 1991. Procesos cognitivos en la comprension de la Ciencia: las ideas de los adolescentes sobre la Química.(CIDE: Madrid).

QUILLEZ, J., 1988. Problemas de Química de COU y Selectividad. (Bello: Valencia).

SAUNDERS, W.J., 1992. The Constructivist Perspective: Implications and Teaching Strategies for Science, School Science and Mathematics, Vol. 92 (3), pp. 136-141.

SELVARATNAM, M. y FRAZER, M.J., 1982.Problem Solving in Chemistry, (Heinemann: Londres).

SELVARATNAM, M. y KUMARASINGHE, S., 1991. Student Conceptions and Competence Concerning Quantitative Relationships between Variables, J. Chem. Ed., Vol. 68 (5), pp. 370-372.

SHAYER, M. y ADEY, P., 1984. La ciencia de enseñar ciencias. Desarrollo cognoscitivo yexigencias del currículo. (Narcea: Mad́rid).

SOLAZ, J.J., 1993. Pourquoi Continuer à Aprendre le Principe de Le Chatelier? Enviado al Bulletin de l'Union des Physiciens.

SOLOMON, T., 1991. The Temperature and Pressure Dependence of the Equilibrium Properties of a System, J. Chem. Ed., Vol. 68 (4), pp. 294-295.

TREPTOW, R.S., 1980. LeChatelier's Principle. A reexamination and method of graphic illustration, $J$. Chem. Ed., Vol. 57 (6), pp. 417-420.

TREPTOW, R.S., 1984. Le Chatelier's Principle Applied to the Temperature Dependence of Solubility, J. Chem. Ed., Vol. 61 (6), pp. 499-502.

WHEELER, A.E. y KASS, H., 1978. Students' misconceptions in chemical equilibrium, Science Education, Vol. 62, pp. 223-232.

WRIGHT, P.G., 1969. A Chatelerian Infidelity, Ed. Chem., Vol. 6, pp. 9 y 18. 